\title{
Importancia de la educación ambiental en la gestión del riesgo socio-natural en cinco países de América Latina y el Caribe
}

\author{
Importance of Environmental Education in Socio-Natural Risk Management in Five \\ Countries of Latin America and the Caribbean
}

\section{A importância da educação ambiental na gestão de risco sócio natural, em cinco países da América Latina e do Caribe}

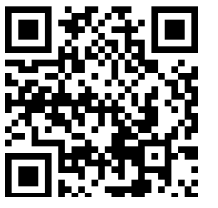

\footnotetext{
${ }^{1}$ Ingeniera Ambiental, Magister en Gestión Ambiental y estudiante de Doctorado en Educación, experiencia como docente a nivel de pregrado y posgrado, como asesora de proyectos de investigación y en actividades académicoadministrativas en el diseño y actualización curricular, en procesos de registro calificado, autoevaluación y proyección social. Docente investigadora en el grupo de investigación SYRAS del programa de ingeniería ambiental de la Universidad Manuela Beltrán.

2 Ingeniera Civil, especialista en Evaluación Ambiental de Proyectos. Cuenta con experiencia laboral en la interventoría de proyectos de infraestructura vial y experiencia investigativa como joven investigadora en el grupo CIDERA desarrollando el proyecto "Inventario de la oferta y demanda hídrica superficial en la Cuenca del Río la Vieja, en el marco del macroproyecto "Software del Inventario de Oferta y Demanda Hídrica superficial del departamento del Quindío".

${ }^{3}$ Máster en Administración de Empresas con Especialidad en Gestión Integrada de la Calidad, Seguridad y Medio Ambiente; estudios de Maestría en Economía Ambiental y de los Recursos Naturales. Experiencia en sector público, privado y organizaciones no gubernamentales (ONG), en funciones administrativas de coordinación de equipo, elaboración y ejecución de proyectos, programas estatales, actividades comunitarias, capacitación y docencia.
}

Martha Melizza Ordóñez-Diaz, Luisa Maria Montes-Arias y Giovanna del Pilar Garzón-Cortés 
doi: http://dx.doi.org/10.15359/ree.22-1.17

URL: http://www.una.ac.cr/educare

CORREO: educare@una.cr

Recibido • Received • Recebido: 12 / 12 / 2016

Corregido • Revised • Revisado: 25 / 11 / 2017

Aceptado • Accepted • Aprovado: 14 / 12 / 2017

Resumen: Considerando la educación ambiental como una herramienta social que permite a los individuos lograr un conocimiento significativo del entorno habitado, disminuir la probabilidad de ocurrencia de un desastre y dar respuesta ante la presencia de los fenómenos naturales a los cuales son vulnerables, el presente artículo tiene como objetivo, generar un espacio de reflexión sobre la importancia de la educación ambiental en la gestión del riesgo socio-natural en cinco países de América Latina y el Caribe, para lo cual se presenta una revisión descriptiva de fuentes bibliográficas primarias y secundarias referentes al desempeño de la gestión del riesgo socio-natural relacionado con la educación ambiental en Colombia, Nicaragua, México, Chile y Jamaica entre 1994 y 2015, periodo en el cual se evidencia una sólida organización administrativa y legislativa respecto a estos dos temas, pero una clara separación de estos mismos en al momento de la implementación de proyectos ciudadanos; situación que ha generado falencias en la gestión de desastres naturales, específicamente desde los principios de precaución y prevención, para lo cual en el presente artículo se establecieron una serie de recomendaciones que incluyen la divulgación de información, la creación de centros para para la gestión de la reducción del riesgo, el fortalecimiento de estrategias de comunicación, el establecimiento de planes de respuesta y recuperación post desastre.

Palabras claves: Ordenamiento territorial; educación ambiental; gestión del riesgo; riesgo socionatural; riesgo en América Latina y el Caribe.

Abstract: Considering environmental education as a social tool allowing individuals to achieve a significant knowledge of the inhabited environment, to reduce the probability of occurrence of a disaster, and to respond to the presence of natural phenomena to which people are vulnerable, this article aims to generate a space for reflection on the importance of environmental education in the management of the social and natural risk in five countries of Latin America and the Caribbean. For this purpose, the paper presents a descriptive review of primary and secondary bibliographical sources referring to the performance of the management of social and natural risks related to environmental education in Colombia, Nicaragua, Mexico, Chile, and Jamaica between 1994 and 2015. In this period, a solid administrative and legislative organization of this management and environmental education is evident, but these two themes are clearly separated when implementing citizen projects: a situation that has generated shortcomings in the management of natural disasters, specifically under the principles of precaution and prevention. For this reason, this article offers a series of recommendations that include the dissemination of information, the creation of centers for the management of risk reduction, the strengthening of communication strategies, and the establishment of response plans and post-disaster recovery.

Keywords: Land use planning; environmental education; management of the risk; social and natural risk; risk in Latin America and the Caribbean. 
Resumo: Considerando a educação ambiental como uma ferramenta social que permite aos indivíduos alcançar um conhecimento significativo do ambiente habitado, esta es capaz de reduzir a probabilidade de ocorrência de um desastre e também responder à presença de fenômenos naturais aos que são vulneráveis. O objetivo deste artigo é criar um espaço de reflexão sobre a importância da educação ambiental na gestão de risco sócio natural em cinco países da América Latina e do Caribe, cuja revisão descritiva de fontes bibliográficas primárias e secundárias, referentes ao desempenho da gestão de risco sócio natural, relacionados com a educação ambiental na Colômbia, Nicarágua, México, Chile e Jamaica, entre 1994 e 2015. É um período em que existem evidências de uma sólida organização administrativa e legislativa em relação a estes dois temas, com uma clara separação de cada um, no momento da execução dos projetos urbanos; situação que levou a deficiências na gestão de desastres naturais, especificamente a partir dos princípios da precaução e da prevenção. Este artigo estabelece uma série de recomendações, incluindo a divulgação de informações, criação de centros de gestão para a redução do risco, fortalecimento das estratégias de comunicação, estabelecendo planos de resposta e de recuperação pós-desastre.

Palavras-chave: ordenação territorial, educação ambiental, gestão de risco, risco socio-natural; risco na América Latina e no Caribe.

\section{Introducción}

Tradicionalmente los programas de mitigación del riesgo llevados a cabo por gobiernos, agencias y ONG, "sólo buscan mitigar las consecuencias de fenómenos naturales específicos y no reducir la vulnerabilidad. Como tal, sólo atacan los síntomas y no las causas de los desastres" (Maskrey, 1989, p. 12); esto se evidencia en la reacción de las personas al enfrentarse a un fenómeno natural, cuando manifiestan que contaban con poca o nula información sobre los riesgos de tipo socio natural a los que están vulnerables y que, por ende, no estaban preparadas para afrontar lo sucedido, tal y como lo menciona Robert Glasser, representante especial de las Naciones Unidas para la reducción del riesgo de resastres:

Encuentro realmente escandaloso e inaceptable que nosotros, exceptuando Haití, hayamos podido ver por televisión como se acercaba la tempestad, mientras que era imposible avisar a la gente del lugar con alertas precoces, o, cuando se mandaban alertas precoces, no servían de nada por la falta de formación. (Redacción El Tiempo, 2016, párr. 3)

Actualmente, América Latina y el Caribe es una región "especialmente vulnerable a los efectos del cambio climático debido a su situación geográfica y climática, su condición socioeconómica y demográfica y la alta sensibilidad al clima de sus activos naturales, como los bosques y la biodiversidad" (Naciones Unidas, 2015, p. 9), situación que, junto con los fenómenos ambientales históricos, aumenta las condiciones de riesgo de desastres naturales e impacta negativamente el bienestar de las personas, el crecimiento económico de la región y la conservación de los recursos naturales. De acuerdo con estadísticas reportadas en Emergency 
doi: http://dx.doi.org/10.15359/ree.22-1.17

URL: http://www.una.ac.cr/educare

CORREO: educare@una.cr

Events Database EM DAT (citado por Ashwill \& Alvarez, 2014), la frecuencia de los desastres naturales, tales como inundaciones, sequías, huracanes y terremotos, está aumentando tanto en América Latina y el Caribe (ALC) como en el resto del mundo. Durante las décadas de 1960 y 1970, menos de 20 desastres ocurrieron por año. Durante los años 2000, el promedio aumentó a 50 desastres por año. Aunque se están volviendo menos mortales, están resultando más costosos en cuanto a los daños totales que implican.

De acuerdo con lo anterior, el propósito del presente artículo es generar un espacio de reflexión sobre la importancia de la educación ambiental en la gestión de riesgo socionatural, para ello, se presenta inicialmente una revisión bibliográfica que apoya la unificación de conceptos relacionados con desastre natural, gestión del riesgo socio-natural, amenaza, vulnerabilidad y educación ambiental; posteriormente se estudia información de fuentes primarias y secundarias que evidencien el desempeño en la gestión del riesgo de desastres y avances de la educación ambiental en cinco países de América Latina y el Caribe (Colombia, Nicaragua, México, Chile y Jamaica), para cada uno de los países seleccionados y de acuerdo con la información disponible de estudios realizados por organizaciones como la Comisión Económica para América Latina y el Caribe (CEPAL), las Naciones Unidas para la Reducción del Riesgo de Desastres y los diferentes entes gubernamentales encargados de la gestión del riesgo que, en ocasiones, es limitada, se presenta una caracterización que incluyó ubicación geográfica, condiciones de riesgo, marco normativo de la gestión del riesgo, educación ambiental y avances en la gestión del riesgo relacionados con la educación ambiental en cada país. Esta información permite plantear al finalizar el artículo estrategias en pro del fomento de la educación ambiental como una herramienta que promueve, en cada individuo, el conocimiento de las condiciones de su entorno y cómo actuar ante la presencia de determinadas situaciones naturales, teniendo en cuenta los resultados del diagnóstico, la vinculación de estos en la etapa de planeación y la creación de planes de acción que deben ser comunicados e informados a todos los actores implicados en la gestión del riesgo socio-natural.

\section{Descripción de la gestión del riesgo socio-natural}

El ordenamiento territorial -OT- considera el tema base de la gestión del riesgo, ya que se relaciona con las directrices y acciones que se consideran desde distintos entes de control social para ordenar el territorio en pro del bienestar de las personas y el desarrollo de una región. En Colombia, la ley 388 de 1997, Artículo $6^{\circ}$, establece que el objeto del ordenamiento territorial es "complementar la planificación económica y social con la dimensión territorial, racionalizar las intervenciones sobre el territorio y orientar su desarrollo y aprovechamiento sostenible" (Congreso de la República de Colombia. Secretaría Jurídica Distrital de la Alcaldía Mayor de Bogotá D. C., 1997). Sin embargo, y en contraposición con las definiciones expuestas, las comunidades indígenas consideran que "el territorio que le fue entregado a cada cultura

\begin{tabular}{l|l}
\hline 4 & Martha Melizza Ordóñez-Diaz, Luisa Maria Montes-Arias y Giovanna del Pilar Garzón-Cortés
\end{tabular}

Los artículos de la Revista Electrónica Educare del Centro de Investigación y Docencia en Educación de la Universidad Nacional, Costa Rica, se comparten bajo términos de la Licencia Creative Commons: Reconocimiento, № Comercial, Sin Obra Derivada 3.0 Costa Rica. Las autorizaciones adicionales a las aquí delimitadas se pueden obtener en el correo: educare@una.cr 
vino con un orden; los ancestros recibieron todas las indicaciones de cómo vivir allí, de cómo manejar ese mundo y ser parte integral de él" (Rivera, 2007, p. 116), de tal forma que debe ordenarse la actividad humana sobre este mismo, afirmación que no es descabellada, ya que, evidentemente, la falta de organización y de interpretación de los fenómenos naturales por parte del ser humano ha conllevado al asentamiento de comunidades humanas en zonas de alto riesgo de desastre, es decir, en territorios marcados por la vulnerabilidad a la ocurrencia de un desastre natural, de acuerdo con la Ley 1523 del 2012, artículo 4 , definición 8:

[Un desastre] es el resultado que se desencadena de la manifestación de uno o varios eventos naturales o antropogénicos no intencionales que al encontrar condiciones propicias de vulnerabilidad en las personas, los bienes, la infraestructura, los medios de subsistencia, la prestación de servicios o los recursos ambientales, causa daños o pérdidas humanas, materiales, económicas o ambientales, generando una alteración intensa, grave y extendida en las condiciones normales de funcionamiento de la sociedad, que exige del Estado y del sistema nacional ejecutar acciones de respuesta a la emergencia, rehabilitación y reconstrucción. (Congreso de la República de Colombia, 2012a, p. 6)

Debido a la vulnerabilidad del ser humano, la cual hace referencia a la predisposición de pérdidas o daños de su integridad, su entorno físico, social o económico por la ocurrencia de un evento natural, desde 1992 diferentes grupos científicos se han interesado en comprender y analizar los desastres naturales, sentando las bases de lo que actualmente se conoce como la gestión del riesgo, definida por Wilches Chaux (1998, citado por Lavell, 2002) como "el proceso a través del cual una sociedad, o subconjuntos de una sociedad, influyen positivamente en los niveles de riesgo que sufren, o podrían sufrir" (p. 2).

Actualmente la gestión del riesgo representa una de las principales herramientas con las que cuenta la sociedad para prevenir los desastres socio-naturales, dentro de la cual es necesario el diseño e implementación de planes de ordenamiento territorial, mapas de riesgo o de peligro actualizados y políticas públicas que involucren: la identificación del riesgo a través de la percepción individual, la representación social y desde el principio de precaución; la reducción del riesgo teniendo en cuenta la prevención, mitigación y la preparación antes de la ocurrencia del desastre y, finalmente, la respuesta y recuperación, es decir, la reconstrucción y rehabilitación de las condiciones de vida de la población afectada Cardona (2001).

Es preciso señalar que la efectividad de la respuesta tanto de organismos como de la población en general depende de los procesos de sensibilización y educación ante el riesgo de desastres y el fomento de hábitos de prevención, ya que representa respuestas positivas por parte de los actores vulnerables frente la ocurrencia del fenómeno, por lo tanto y apoyando la afirmación de Reyes (2012), es indispensable que las organizaciones sociales y el sector educativo 
doi: http://dx.doi.org/10.15359/ree.22-1.17

URL: http://www.una.ac.cr/educare

CORREO: educare@una.cr

asuman la gestión del riesgo ante desastres socio-naturales como parte de los procesos de reflexión, educación y transformación en pro de causar en la comunidad un nuevo sentir, pensar y actuar. De acuerdo con lo planteado por Ferrer, Menéndez y Gutiérrez (2004, citados por Miranda, 2013), "únicamente a través de la educación como el individuo interioriza la cultura, y es capaz de construir y producir conocimientos, reorientar sus valores, modificar sus acciones y contribuir como sujeto individual a la transformación de la realidad del medio ambiente" (p. 96).

Algunas de las conclusiones presentadas por Cardona (2008) en el último informe de la Medición de la gestión del riesgo en América Latina permiten observar que en la región hace falta mejorar los escenarios de daños y pérdidas potenciales de todas las ciudades, contar con mapas de amenaza a nivel subnacional y municipal, ampliar el grado de información pública y de participación de la comunidad en los procesos de percepción y representación del riesgo. Un reflejo de las falencias a nivel regional se evidencia en las incalculables pérdidas acumuladas por desastres naturales y que permiten generar una visión acerca del nivel de riesgo a que se encuentra expuesta América Latina. A continuación, se mencionan cifras al respecto publicadas por la Oficina de las Naciones Unidas para la Reducción del Riesgo de Desastres (UNISDR, 2013):

En el conjunto de los 16 países [de Latinoamérica], Colombia y México, [cuentan] con las poblaciones más altas, y tienen la mayor cantidad de registros ( $45 \%$ del total), de personas afectadas (64\%) y viviendas dañadas (70\%). Así mismo, Honduras y Colombia, concentran más de la mitad de la pérdida de vidas humanas (59\%)...

Entre 1990 y 2011 se registraron más de 16 mil personas fallecidas por manifestaciones intensivas del riesgo asociadas con eventos hidrometeorológicos y climáticos; de éstas, 12 770, que representan el 79\% del total, ocurrieron en 4 países: México, Nicaragua, Honduras y Venezuela.

Chile, Perú, Nicaragua y Jamaica (...) hacen parte de los países que registran mayores cantidades de daños y pérdidas. En todos los casos, se trata de impactos acumulados espacial y temporalmente por eventos hidrometeorológicos y climáticos, en algunos casos, extremos. (pp. 28, 37, 47)

\section{Incidencia de la educación ambiental en la gestión del riesgo socio-natural en América Latina y el Caribe}

El entendimiento del riesgo implica tanto el conocimiento de la amenaza como de la vulnerabilidad y su gestión adecuada involucra una apropiada transmisión de información respecto a la amenaza y adecuados procesos educativos que permitan la reducción de la vulnerabilidad. La educación ambiental es: 
Artículo $1^{\circ}$. Un proceso dinámico y participativo, orientado a la formación de personas críticas y reflexivas, con capacidades para comprender las problemáticas ambientales de sus contextos (locales, regionales y nacionales). Al igual que para participar activamente en la construcción de apuestas integrales (técnicas, políticas, pedagógicas y otras), que apunten a la transformación de su realidad, en función del propósito de construcción de sociedades ambientalmente sustentables y socialmente justas. (Congreso de la República de Colombia, 2012b, párr. 1)

De acuerdo con lo anterior, la educación ambiental se presenta como un instrumento de acción social frente al manejo de los desastres naturales, que permite a cada individuo conocer y comprender las características del entorno habitado y adquirir la capacidad de actuar en la disminución de la probabilidad de ocurrencia de un desastre y en la respuesta adecuada ante la presencia de los fenómenos naturales a los cuales son vulnerables; como lo expone Lavell (1999) "la clave de la reducción del riesgo está en el entendimiento del riesgo mismo, en la educación acerca de él, y en la participación decidida y comprometida de todos los actores sociales, privados y públicos, en su resolución" (p. 12). Es preciso mencionar que la predisposición de un individuo ante un evento calamitoso varía de acuerdo con la preparación que este tenga para enfrentar dicho evento y la no preparación de dicho individuo acompañado de la predisposición del espacio geográfico ante la ocurrencia de un fenómeno natural, lo convierten en un elemento vulnerable.

Como se puede evidenciar, la educación ambiental es una estrategia para el entendimiento del riesgo, que involucra el conocimiento de la amenaza y de la vulnerabilidad y cuya gestión del riesgo promueve el transmitir y compartir información respecto a la amenaza y adecuados procesos de actuación que permitan su reducción.

Comprender que hay elementos significativos causados por el ser humano en todos los tipos de desastre y que se necesita más conciencia en cuanto a las diversas opciones con que se cuenta para prevenir los desastres, "[apunta] hacia la necesidad de fortalecer el enfoque de la concientización pública y en [promover la] educación ambiental para ayudar a reducir el riesgo de desastres" (Federación Internacional de Sociedades de la Cruz Roja y de la Media Luna Roja, 2011, p. 7), específicamente en las etapas anteriores al desastre que son las etapas en las cuales se conoce el entorno, se identifica la amenaza y se realizan las labores de mitigación y de prevención del riesgo.

A continuación, se describen los avances en materia de gestión del riesgo socio natural relacionados con la educación ambiental, de cinco países de América Latina y el Caribe, seleccionados por su representatividad en el último informe realizado por la UNISDR (2013), en el cual se destacan los siguientes datos, en el marco de objeto de estudio del presente artículo, los cuales son de interés de las autoras: "Nicaragua, [el país más grande de los localizados en el Istmo Centroamericano, está] junto con México, El Salvador, y Colombia, dentro de los 5 países con mayor cantidad de pérdida de vidas humanas entre 1990 y 2011 " (p. 35). 
doi: http://dx.doi.org/10.15359/ree.22-1.17

URL: http://www.una.ac.cr/educare

CORREO: educare@una.cr

"Después de México, Chile fue el segundo país en el 2010 con mayores daños y pérdidas totales debido [al último] sismo del 27 de febrero de ese año, mientras que en 2011 fue el octavo país" (p. 18); se torna conveniente por medio del presente estudio analizar las medidas en gestión del riesgo implementadas en dicho país para desplazarse del segundo al octavo lugar en un año.

Jamaica, un país pequeño en territorio y en cantidad de población, pero históricamente expuesto al recorrido de los huracanes durante su paso por el Caribe, por tanto, en dicho territorio, el desarrollo de políticas, acciones e inversiones de reducción de riesgos resultan prioritarias.

\section{- Nicaragua}

Es un país ubicado en el centro del istmo centroamericano. Limita al norte con Honduras, al sur con Costa Rica, al oeste con el océano Pacífico y al este con el mar Caribe. "Los siete fenómenos naturales con mayor potencial de impacto en Nicaragua son: sismos, tsunamis, vulcanismo, huracanes, sequías, movimientos de laderas e inundaciones"(Banco Interamericano de Desarrollo [BID], Naciones Unidas [UN] y Comisión Económica para América Latina y el Caribe [CEPAL], 2007b, p. 21). “La topografía montañosa del país, que abarca 21 cuencas hidrográficas, contribuye a su alta vulnerabilidad a este tipo de amenazas naturales" (Banco Interamericado de Desarrollo, 2010, párr. 3). En Nicaragua se cuenta con el Sistema Nacional de Prevención, Mitigación y Atención de Desastres (SINAPRED) y en materia de educación ambiental con el Ministerio del Ambiente y los Recursos Naturales (MARENA) y la Comisión Nacional de Educación Ambiental (CNEA). El documento rector de la educación ambiental se titula Lineamientos de política y estrategia nacional de educación ambiental, cuyos principales objetivos son: "promover en la población nicaragüense la sensibilización y toma de conciencia con respecto a su entorno ambiental, los problemas que en él se manifiestan, sus causas y consecuencias, así como las posibles alternativas de solución y prevención de los mismos" (Ministerio del Ambiente y los Recursos Naturales [MARENA], 2004, p. 13). Dentro de los avances más significativos en gestión del riesgo relacionadas con la educación ambiental en Nicaragua se destacan: La Ley N. ${ }^{\circ} 311$ de 1981: Ley Orgánica del Instituto Nicaragüense de Estudios Territoriales.

[Esta] atribuye las responsabilidades y competencias al INETER como el órgano encargado de varias tareas: de la investigación, inventario y evaluación de los recursos físicos del país y de ejecutar los estudios de ordenamiento territorial; de realizar los estudios para la prevención y mitigación de los efectos provocados por fenómenos naturales peligroso; de realizar los estudios meteorológicos y geofísicos; de regular y efectuar los trabajos cartográficos y geodésicos y de normar, regular, operar, actualizar y ejecutar el catastro físico nacional (Oficina de las Naciones Unidas para la Reducción del Riesgo de Desastres [UNISDR] y Centro de Coordinación para la Prevención de los Desastres Naturales en América Central [CEPREDENAC], 2013, p. 20, fila 12, columna 2).

8 Martha Melizza Ordóñez-Diaz, Luisa Maria Montes-Arias y Giovanna del Pilar Garzón-Cortés

Los artículos de la Revista Electrónica Educare del Centro de Investigación y Docencia en Educación de la Universidad Nacional, Costa Rica, se comparten bajo términos de la Licencia Creative Commons: Reconocimiento, № Comercial, Sin Obra Derivada 3.0 Costa Rica. Las autorizaciones adicionales a las aquí delimitadas se pueden obtener en el correo: educare@una.cr 
[La capacitación] a los miembros integrantes de la Comisión de Trabajo Sectorial de Educación e Información [y docentes de educación primaria, secundaria y universitaria] en la temática de seguridad escolar, administración de albergues y normas de la Red Internacional para la Educación en Situaciones de Emergencia ... [y reducción de riesgo de desastres]; [Se han] experimentado avances en la implementación de programas de sensibilización y de educación sobre RRD [Reducción de Riesgo de Desastre], así como en la capacitación específica destinada a funcionarios/as y a líderes comunitarios; ... El tema de la comunicación se vincula con la educación, de ahí que el país cuenta con una Estrategia Nacional de Comunicación para la Gestión Integral de Riegos ..., [que] promueve la generación, el intercambio y la utilización de la información para lograr una población informada, mejor preparada y generadora de una cultura de prevención. (UNISDR y CEPREDENAC, 2013, pp. 49, 121, 179):

\section{- México}

Limita al norte con Estados Unidos, al sur con Guatemala y Belice, al occidente con el Océano Pacifico y al oriente con el Golfo de México. Debido a su ubicación, México está expuesto a la ocurrencia de desastres naturales como sismos, inundaciones, sequías y heladas. Como eje central de la política relacionada con la gestión de riesgo de desastres (GRD), existe el Sistema Nacional de Protección Civil (SINAPROC), creado en 1986 a raíz de los terremotos sufridos en el país durante septiembre de 1985, cuyo objetivo es coordinar las acciones y las instituciones relacionadas con la prevención y atención de desastres y se respalda en la Ley General de Protección Civil aprobada en el 2000 a nivel nacional y las Leyes Estatales aprobadas en cada una de las entidades federativas, "estos instrumentos son de carácter normativo y se sustentan en la noción de preparativos y respuesta, así como en la coordinación interinstitucional para tales fines" (Mansilla, 2008, p. 31).

La institución gubernamental encargada de las iniciativas en educación ambiental es el Centro de Educación y Capacitación para el Desarrollo Sustentable (CECADESU) de la Secretaría de Medio Ambiente y Recursos Naturales (SEMARNAT).

México, a través de la Secretaría de Medio Ambiente y Recursos Naturales (SEMARNAT) y de la Secretaria de Educación Pública (SEP) ... suscribieron, en marzo del año 2005, un Compromiso Nacional por el Decenio de la Educación para el Desarrollo Sostenible. Siendo el primer país en América Latina y el Caribe en implementar acciones en el marco del decenio. (OREALC/UNESCO, 2009, p. 60)

La misión de esta estrategia es establecer políticas públicas en el plano nacional y local en educación ambiental para la sustentabilidad "que favorezcan la construcción de una cultura ambiental, el incremento en la calidad de vida de la población, el fortalecimiento de la 
doi: http://dx.doi.org/10.15359/ree.22-1.17

URL: http://www.una.ac.cr/educare

CORREO: educare@una.cr

ciudadanía y de las múltiples identidades culturales del país, y la protección de los ecosistemas y su biodiversidad" (Secretaría de Medio Ambiente y Recursos Naturales, SERMANAT, 2006 p. 208); en este sentido, dentro de los avances más significativos en gestión del riesgo relacionadas con la educación ambiental en México se destaca: la Ley general de protección civil vigente desde el 2012, art.43, punto III, la cual establece como uno de los deberes de las autoridades nacionales"concretar el establecimiento de programas educativos a diferentes niveles académicos, que aborden en su amplitud el tema de la protección civil y la Gestión Integral de Riesgos" (Congreso General de los Estados Unidos Mexicanos, 2012, p. 20), la cual dio paso a la creación de la Coordinación General de Protección Civil en 2014, con el objetivo de liderar procesos de cultura de protección civil, a través de capacitaciones, caravanas de prevención y simulacros, "dirigidas a la población en general, con la finalidad de que le permita conocer de forma clara lo [sic] mecanismos de prevención y autoprotección ante una calamidad" (Coordinación General de Protección Civil, Gobierno del Estado de México, 2014, párr. 1). “La Secretaría de Educación Pública (SEP) después de los sismos de septiembre del 1985, dio inicio a un programa [obligatorio y] permanente de simulacros en las escuelas oficiales de enseñanza básica" (BID, UN, CEPAL, 2007a, p. 178).

\section{- Chile}

Se ubica en el extremo sudoeste de América del Sur, limita al sur con el Paso Drake, al este con Bolivia y Argentina y al oeste con el Océano Pacífico. Cada región chilena tiene sus propios riesgos, por ejemplo, sismos o terremotos, incendios, aluviones, erupciones volcánicas, inundaciones, tsunamis (Educarchile y Energía Andina, 2014). A nivel nacional, la Oficina Nacional de Emergencia ONEMI, a partir del Decreto Ley 369 de 1974, es la institución encargada de la coordinación del Sistema Nacional de Protección Civil, la cual diseña y ejecuta medidas de prevención, respuesta y rehabilitación frente a desastres de origen natural o antrópicos. En Chile la institución gubernamental encargada de las iniciativas nacionales en educación para el desarrollo sostenible es la Comisión Nacional del Medio Ambiente (CONAMA) y Ministerio de Educación (MINEDUC). Dentro de las fortalezas con que cuenta Chile para reducir el nivel de riesgo, teniendo en cuenta la educación ambiental se encuentra la Política Nacional de Educación para el Desarrollo Sustentable (PNEDS), publicada en el 2009, cuyo objetivo es "formar personas y ciudadanos/as capaces de asumir individual y colectivamente la responsabilidad de crear y disfrutar de una sociedad sustentable [y contribuir al] fortalecimiento de procesos educativos que [permitan instalar y desarrollar] valores, conceptos, habilidades, competencias y actitudes en la ciudadanía en su conjunto" (Ministerio del Medio Ambiente, Gobierno de Chile, 2015, p. 13).

Además, en el 2010 selanzó el programa ChilePreparado, el cual busca a través de simulacros promover entre la comunidad la prevención y autocuidado frente a fenómenos naturales. En los últimos años se han llevado a cabo múltiples campañas de educación respecto de amenazas y formas de protegerse como el Plan Familia Preparada. Asimismo, se está capacitando a medios de comunicación para transmitir información sobre alertas y emergencias, a establecimientos

\footnotetext{
10 Martha Melizza Ordónez-Diaz, Luisa Maria Montes-Arias y Giovanna del Pilar Garzón-Cortés
}

Los artículos de la Revista Electrónica Educare del Centro de Investigación y Docencia en Educación de la Universidad Nacional, Costa Rica, se comparten bajo términos de la Licencia Creative Commons: Reconocimiento, № Comercial, Sin Obra Derivada 3.0 Costa Rica. Las autorizaciones adicionales a las aquí delimitadas se pueden obtener en el correo: educare@una.cr 
educacionales para la elaboración de sus respectivos planes integrales de seguridad escolar y a miembros y autoridades del Sistema Nacional de Protección Civil, que se lleva a cabo principalmente a través de la Academia de Protección Civil, lo anterior se complementa con capacitaciones en línea y ejercicios presenciales. (Organización de las Naciones Unidas para la Educación, la Ciencia y la Cultura [Unesco], 2012).

\section{- Jamaica}

Es una isla, situada en el mar Caribe, al oeste se encuentra Centroamérica a $630 \mathrm{~km}$, al norte Cuba a 150 km y a 180 km de la isla La Española al este. "Los fenómenos naturales cuya amenaza tiene la mayor importancia para el país son el terremoto y el huracán, seguidos por inundaciones y deslizamientos" (BID-CEPAL-IDEA, 2004, p. 5). La institución encargada de la gestión del riesgo de desastres en Jamaica es la Oficina de Preparación para Desastres y Gestión de Emergencias (ODPEM). Jamaica en el año 1998, a través del NEEC -Comité Nacional de Educación Ambientalpublicó el Plan de Acción Nacional de Educación Ambiental para el Desarrollo Sostenible, cuyo objetivo es formar, a través de su sistema educativo y de las modalidades formales y no formales, una ciudadanía consciente y comprometida con la protección del medio ambiente y la consecución y mantenimiento de un desarrollo sostenible en Jamaica.

Este plan es un marco de acción nacional para incorporar la educación ambiental en todos los aspectos de la vida de Jamaica, por ejemplo, en el informe Gestión del Riesgo de Desastres elaborado dentro del marco del Programa de las Naciones Unidas para el Desarrollo se destacan: Iniciativas para la incorporación de la gestión del riesgo de desastres en las políticas públicas y para la planificación de la recuperación (desarrollo de centros de gestión para la reducción de riego); la implementación de procesos de recuperación pos desastre (programas de apoyo en la recuperación tras la ocurrencia de huracanes); El Plan Nacional de Desastres, que establece la mitigación, preparación, respuesta y los procedimientos de recuperación para una variedad de riesgos, tanto naturales como provocados por el ser humano; la conciencia pública y la educación son aspectos importantes incluidos en el programa nacional de gestión de riesgos de Jamaica (Progama de las Naciones Unidas para el Desarrollo [PNUD], 2014).

\section{- Colombia}

Se localiza al noroeste del continente de América del Sur. Al norte limita con Panamá y el mar Caribe, por el Oriente con Venezuela y Brasil, por el sur con Perú y Ecuador, y por el occidente con el Océano Pacífico. "Colombia está constituida por una amplia diversidad geológica, geomorfológica, hidrológica y climática, la cual se expresa en un conjunto de fenómenos que representan una potencial amenaza para el desarrollo social y económico del país" (Campos, Corporación OSSO, Rubiano, Díaz y Costa, 2012, p. 13), como fenómenos del niño y la niña, movimientos en masa, erupciones volcánicas y sismos. 
doi: http://dx.doi.org/10.15359/ree.22-1.17

URL: http://www.una.ac.cr/educare

CORREO: educare@una.cr

En Colombia la gestión del riesgo se implementa a partir de 1988 con el Sistema Nacional para la Prevención y Atención de Desastres (SNPAD), ente encargado de la elaboración e implementación de los planes de contingencia a nivel nacional; en el año 2012 se establece la Política Nacional de Gestión del Riesgo de Desastres, Ley 1523 y el Sistema Nacional de Gestión del Riesgo de Desastres (Congreso de la República de Colombia, 2012a), por su parte, la institución gubernamental encargada de las iniciativas nacionales en educación ambiental es el Ministerio de Educación Nacional (MEN) en conjunto con el Ministerio de Ambiente y Desarrollo Sostenible (MADS), los cuales crearon, desde el 2002, la Política Nacional de Educación Ambiental, cuyo fortalecimiento institucional se efectúa a partir de la Ley 1549 del 5 de julio de 2012 (Congreso de la República de Colombia (2012b) y se incorpora efectivamente en el desarrollo territorial.

Dentro de los avances más significativos en gestión del riesgo relacionadas con la educación ambiental en Colombia se destacan: la Política de ordenamiento territorial en Colombia, la cual "ha incorporado funciones de gestión de riesgos con criterios de sostenibilidad, equilibrio territorial y con cohesión institucional, a través de una formulación de planes y proyectos" (Martínez, 2010, p. 292); El Plan Nacional de Gestión del Riesgo de Desastres de Colombia, adoptado en el año 2016, con el cual se pretende "implementar acciones conjuntas para llevar a cabo el proceso social de la gestión del riesgo, contribuyendo a la seguridad, al mejoramiento de la calidad de vida y al desarrollo sostenible" (Unidad Nacional para la Gestión de Riesgo de Desastres, 2016, p. 7).

Sin embargo, es importante mencionar que en Colombia, "a nivel nacional se cuenta con una normatividad extensa y variada en lo referente a la gestión del riesgo [socio-natural], pero se carece de un mecanismo vinculante, ... con aspectos de seguimiento y monitoreo que viabilice su sustentabilidad" (Luengas, 2008, p. 7), además "existen pocos estudios de riesgo y los que existen son muy recientes, [se cuenta con] estudios de amenaza, muchos ... denominado estudios de riesgo en forma equivocada, pues sólo hacen referencia al fenómeno y no tienen en cuenta la vulnerabilidad" (BID, UN, CEPAL, 2007c, p. 1).

\section{Recomendaciones}

La integración del enfoque de la gestión del riesgo en el sector educativo es determinante para incrementar la concientización sobre el efecto y causa de los desastres. Las escuelas que incluyen acciones de gestión del riesgo contribuyen a una cultura para la prevención, esencial para el desarrollo sostenible de los países. Estas acciones reducen los riesgos de desastres y fortalecen las capacidades de las comunidades más vulnerables para responder a las emergencias. (OREALC/UNESCO, 2017, párr. 2) 
Por ende, es necesario que la gestión del riesgo este articulada a los procesos educativos, esto se traduce en una mejor respuesta de los individuos ante la ocurrencia de eventos calamitosos. Frente a lo anterior, es preciso mencionar que en la gestión del riesgo socio-natural, la cual implica identificación del riesgo (conocimiento del entorno, de la amenaza), reducción del riesgo (prevención y mitigación) y manejo de desastres (respuesta y recuperación), la educación ambiental juega un papel fundamental. Maldonado (2005) afirma:

La educación ambiental debe reorientarse y enfocarse en términos continuos y permanentes que vaya desde el Preescolar hasta la Educación Superior, con capacidad real de formar un ciudadano capaz de enfrentar y corregir los procesos y eventos (naturales o antrópicos) desestabilizadores del equilibrio ambiental. (p. 61)

Realmente, es importante el diseño e implementación de programas y proyectos apoyados en procesos educativos y de sensibilización, que promuevan cambios de actitud en las personas y les permita identificar, prevenir y actuar ante la ocurrencia de un fenómeno natural, minimizando la vulnerabilidad y afectación socio ambiental.

Teniendo en cuenta lo anterior, las autoras del presente artículo plantean las siguientes estrategias en pro de fomentar la educación ambiental como una herramienta que le permite a cada individuo conocer su entorno y saber cómo actuar ante determinadas situaciones naturales:

- Vincular los resultados del diagnóstico (que incluyen la caracterización de la amenaza y de los factores de vulnerabilidad de la población como lo son la ausencia de conocimiento, la pobreza, la localización en zonas de alto riesgo) a la etapa de planeación de la gestión de riesgo de desastres socionaturales, ya que así se asegura un beneficio de la población al crear acciones en pro de reducir los efectos de los riesgos potenciales.

- Coordinar, con los diferentes actores expuestos, planes de acción elaborados a partir de la planeación de la gestión del riesgo de desastres socionaturales.

- Comunicar, informar y educar a todos los actores expuestos sobre el tipo de amenaza al que están expuestos y sobre el tipo de acciones (planes de acción) que se deben tomar para reducir el riesgo potencial y asegurar la participación de la población expuesta en los programas de comunicación, información y educación sobre estos mismos.

- Integrar la divulgación de información respecto a estudios realizados sobre riesgo y vulnerabilidad a la educación ambiental, en todos los niveles de educación, para crear, así, en cada individuo, una visión real del entorno que lo rodea y los riesgos a los que se encuentra vulnerable. 
- Establecer centros para la gestión de la reducción del riesgo que se encarguen, en conjunto con las instituciones territoriales, del análisis y reducción del peligro y factores de vulnerabilidad, la transmisión adecuada de información respecto gestión del riesgo local y la preparación de la población en las etapas de respuesta y recuperación.

\section{Conclusiones}

En países como Colombia y México la educación ambiental se basa en las problemáticas ambientales globales y la gestión del riesgo se está enfocando en las etapas de rescate, rehabilitación y reconstrucción y no en las etapas de prevención y preparación que son las que encaminan a las personas a actuar adecuadamente en el momento de ocurrencia de los fenómenos. Por el contrario, en Nicaragua, Chile y Jamaica los avances en políticas de gestión del riesgo se fundamentan en el principio de precaución, prevención y autocuidado que se fomenta a través del programa de simulacros.

La educación ambiental en la gestión del riesgo socionatural es fundamental porque aporta conocimiento a los actores sociales vulnerables a la ocurrencia de un fenómeno natural sobre el nivel de riesgo y el tipo de amenaza al que se encuentran expuestos y así mismo les permite actuar antes y después de la ocurrencia del fenómeno natural.

A partir de la información recopilada respecto a la manera en que se están llevando a cabo los procesos de gestión del riesgo en América Latina y el Caribe ,se observa que existe una sólida organización y legislación respecto al tema; sin embargo, dicha organización presenta falencias de ejecución principalmente en la etapa de prevención, que deben ser corregidas para mejorar el proceso de gestión de riesgo socio natural.

En los cinco países es importante destacar la importancia que ha tomado la educación ambiental en el manejo de las problemáticas y desastres ambientales, como se puede evidenciar, cada uno de los países cuenta con políticas de educación ambiental y de gestión del riesgo; sin embargo, no es notable la articulación de estas mismas en los diferentes planes y programas de prevención, mitigación, respuesta y rehabilitación de desastres, lo cual se evidencia en la magnitud del daño social originado por la ocurrencia de los fenómenos naturales, consecuencia del poco conocimiento con el que cuentan las personas sobre los riesgos de tipo socionatural a los que son vulnerables de acuerdo con las características de los lugares que habitan y, por ende, no están preparadas para enfrentar un fenómeno natural.

\section{Referencias}

Ashwill, M., \& Alvarez, L. (2014). Climate change and IDB: Building resilience and reducing emissions. Sector study: Disaster risk reduction. Washington, D.C.: Inter-American Development Bank. 
Recuperado de https://publications.iadb.org/bitstream/handle/11319/6709/BackgroundPaper-Climate-Change-Adaptation-and-Disaster-Risk-Management.pdf?sequence $=1$

Banco Interamericano de Desarrollo. (01 de octubre de 2010). Nicaragua mejorará la gestión de riesgo de desastres y adaptación al cambio climático con apoyo del BID. Banco Interamericano de Desarrollo. Recuperado de http://www.iadb.org/es/noticias/ comunicados-de-prensa/2010-10-01/nicaragua-mejorara-gestion-riesgo-desastres-yadaptacion-cambio-climaticobid,8097.html

Banco Interamericano de Desarrollo, Comisión Económica para América Latina y el Caribe e Instituto de Estudios Ambientales. (2004). Indicadores de riesgo y de gestión del riesgo de los desastres: Resultados para Jamaica. Recuperado de http://idea.unalmzl.edu.co/ documentos/Perfil\%20Jamaica\%20Indicadores\%20BID-IDEA.pdf

Banco Interamericano de Desarrollo, Naciones Unidas y Comisión Económica para América Latina y el Caribe. (2007a). Información para la gestión de riesgo de desastres estudio de caso de cinco países. México. México: CEPAL, BID. Recuperado de http://www.cepal.org/ publicaciones/xml/9/33659/Mexicoliviano.pdf

Banco Interamericano de Desarrollo, Naciones Unidas y Comisión Económica para América Latina y el Caribe. (2007b). Información para la gestión de riesgo de desastres. Estudio de caso de cinco países. Nicaragua. México: CEPAL, BID. Recuperado de https://www.cepal. org/publicaciones/xml/4/33654/nicaragua.pdf

Banco Interamericano de Desarrollo, Naciones Unidas. Comisión Económica para América Latina y el Caribe. (2007c). Información para la gestión de riesgo de desastres. Estudio de caso de cinco países. Colombia. México: CEPAL, BID. Recuperado de http://repositorio.cepal.org/ handle/11362/25930

Campos, A., Corporación OSSO, Rubiano, D. M., Díaz, C. y Costa, C. R. (2012). Comportamiento de riesgo de desastres en Colombia 1. En A. Campos, N. Holm-Nielsen, C. Díaz, D. M. Rubiano, C. R. Costa, F. Ramírez, ... (Coords., Eds.), Análisis de la gestión del riesgo de desastres en Colombia. Un aporte para la construcción de políticas públicas (pp. 11-71). Bogotá: Banco Mundial. Recuperado de http://gestiondelriesgo.gov.co/sigpad/archivos/ GESTIONDELRIESGOWEB.pdf

Cardona, O. D. (Junio, 2001). La necesidad de repensar de manera holística los conceptos de vulnerabilidad y riesgo. "Una crítica y una revisión necesaria para la gestión". Ponencia presentada en International work-conference on vulnerability in disater theroy and practice. Recuperado de http://www.desenredando.org/public/articulos/2003/rmhcvr/rmhcvr may-08-2003.pdf 
doi: http://dx.doi.org/10.15359/ree.22-1.17

URL: http://www.una.ac.cr/educare

CORREO: educare@una.cr

Cardona, O. D. (2008). Medición de la gestión del riesgo en América Latina. Revista Internacional de Sostenibilidad, Tecnología y Humanismo, 3, 1-20. Recuperado de http://upcommons. upc.edu/bitstream/handle/2099/7056/cardona.pdf

Congreso de la República de Colombia. (24 de abril de 2012a). Ley 1523 "Por la cual se adopta la política nacional de gestión de riesgo de desastres y se establece el sistema nacional de gestión del riesgo de desastres y se dictan otras disposiciones". Recuperado de http://www. ideam.gov.co/documents/24189/390483/11.+LEY+1523+DE+2012.pdf/4e93527d-3bb84b53-b678-fbde8107d340?version=1.2

Congreso de la República de Colombia. (5 de julio de 2012b). Ley 1549 "Por medio de la cual se fortalece la institucionalización de la política nacional de educación ambiental y su incorporación efectiva en el desarrollo territorial". Diario Oficial No. 48.482. Recuperado de http://wsp.presidencia.gov.co/Normativa/Leyes/Documents/ley154905072012.pdf

Congreso de la República de Colombia. Secretaría Jurídica Distrital de la Alcaldía Mayor de Bogotá D. C. (1997). Ley 388 de 1997 Nivel Nacional. Recuperado de http://www.alcaldiabogota. gov.co/sisjur/normas/Norma1.jsp?i=339

Congreso General de los Estados Unidos Mexicanos (6 de junio de 2012). Ley general de protección civil. Diario Oficial de la Federación. Recuperado de http://www.ifrc.org/docs/ IDRL/LeyGPC.pdf

Coordinación General de Protección Civil, Gobierno del Estado de México. (2014). Cultura de protección civil. Recuperado de http://cgproteccioncivil.edomex.gob.mx/cultura proteccion civil

Educarchile y Energía Andina. (2014). Ficha temática: Riesgos naturales. Recuperado de http:// www.educarchile.cl/ech/pro/app/detalle?id=223751

Federación Internacional de Sociedades de la Cruz Roja y de la Media Luna Roja. (2011). Guía: Para la sensibilización y educación pública sobre la reducción de riesgos de desastres. Ginebra: Autor. Recuperado de http://www.ifrc.org/Global/Publications/disasters/reducing risks/302200-Public-awareness-DDR-guide-ES.pdf

Lavell, A. (1999). Gestión de riesgos ambientales urbanos. Panamá: Flacso y La Red. Recuperado de http://www.unisdr.org/files/11008 GestionDeRiesgosAmbientalesUrbanos1.pdf

Lavell, A. (2002). Sobre la gestión del riesgo: Apuntes hacía una definición. Recuperado de http:// www.bvsde.paho.org/bvsacd/cd29/riesgo-apuntes.pdf 
Luengas, M. E. (2008). La incorporación de la gestión del riesgo en instituciones educativas del municipio de Los Patios. Lineamientos. San José de Cúcuta: Alcaldía municipal. Recuperado de http://www.comunidadandina.org/predecan/doc/libros/pp/co/PEGR.pdf

Maldonado, H. A. (2005). La educación ambiental como herramienta social. Geoenseñanza, 10 (1), 61-67. Recuperado de http://www.saber.ula.ve/bitstream/123456789/21003/2/ articulo4.pdf

Mansilla, E. (2008). Marco General de Riesgo en México. Recuperado de http://www.grid.unep.ch/ activities/earlywarning/download/GAR draft/Alejandro documents/Mexico/Mexico-H2. pdf

Martínez, M. T. (2010). Perspectiva territorial de la gestión de riesgos de desastres en Colombia. Perspectiva Geográfica, 15, 277-298. Recuperado de http://revistas.uptc.edu.co/index.php/ perspectiva/article/view/1743/1738

Maskrey, A. (1989). El manejo popular de los desastres naturales. Estudios de vulnerabilidad y mitigación. Lima: ITDG. Recuperado de https://www.google.com.co/url?sa=t\&rct=j\&q=\& esrc=s\&source $=$ web \&cd $=1 \& \mathrm{cad}=\mathrm{rja} \&$ uact $=8 \& \mathrm{ved}=0 \mathrm{CBsQFjAA} \& u r l=\mathrm{http} \% 3 \mathrm{~A} \% 2 \mathrm{~F} \% 2 \mathrm{Fw}$ ww.solucionespracticas.org.pe\%2FDescargar\%2F99\%2F492\&ei=HjBIVYLHGsP5ggTrm4P oDw\&usg=AFQjCNENvYiLPopzMJsg7X0hshl0h4ADyQ\&sig2=0uFeMUuLPMzJRBpSFQ

Ministerio del Ambiente y los Recursos Naturales. (2004). Lineamientos de política y estrategia nacional de educación ambiental. Managua: Marena. Recuperado de http://www.bvsde. org.ni/Web textos/MARENA/MARENA0309/0309Politicaeduambientallineamientos.pdf

Ministerio del Medio Ambiente, Gobierno de Chile. (2015). Política nacional de educación para la sustentabilidad (3a ed.). Recuperado de http://media.picalab.cl/sustentabilidad/ curriculo educaci\%C3\%B3n sustentable/Pol\%C3\%ADtica\%20de\%20ES\%20-EDS\%20 versi\%C3\%B3n\%20borrador\%202017.pdf

Miranda, L. M. (2013). Cultura ambiental: Un estudio desde las dimensiones de valor, creencias, actitudes y comportamientos ambientales. Revista Producción + Limpia. 8(2), 94-105. Recuperado de http://www.scielo.org.co/pdf/pml/v8n2/v8n2a10.pdf

Naciones Unidas. (2015).Laeconomía del cambio climático en América LatinayelCaribe.Paradojas y desafíos del desarrollo sostenible. Santiago de Chile: Autor. Recuperado de http:// repositorio.cepal.org/bitstream/handle/11362/37310/S1420656 es.pdf?sequence $=4$

Oficina de las Naciones Unidas para la Reducción del Riesgo de Desastres. (2013). Impacto de los desastres en América Latina y el Caribe, 1990 y 2011. Tendencias y estadísticas para 16 países (informe). Panamá: Autor. Recuperado de http://eird.org/americas/noticias/Impacto de los desastres en las Americas.pdf 
Oficina de las Naciones Unidas para la Reducción del Riesgo de Desastres y Centro de Coordinación para la Prevención de los Desastres Naturales en América Central. (2013). Informe sobre la gestión integral del riesgo de desastres en Nicaragua 2013. Managua: UNISDR. Recuperado de http://eird.org/pr14/cd/documentos/espanol/ AmericaCentralHerramientasydocumentos/Informesregionalynacionales/Informe-GIRDNicaragua-version-web.pdf

OREALC/UNESCO. (2009). Políticas, estrategias y planes regionales, subregionales y nacionales en educación para el desarrollo sostenible y la educación ambiental en América Latina y el Caribe, Decenio de las Naciones Unidas de la Educación para el Desarrollo Sostenible 2005-2014. Santiago: Autor. Recuperado de http://unesdoc.unesco.org/ images/0018/001819/181906S.pdf

OREALC/UNESCO. (2017). Educación para la gestión del riesgo ante desastres. Santiago: Autor. Recuperado de http://www.unesco.org/new/es/santiago/education/disaster-riskmanagement-education/

Organización de las Naciones Unidas para la Educación, la Ciencia y la Cultura. (2012). Análisis de riesgos de desastres en Chile. VII plan de acción dipecho en Sudamérica 2011-2012. Santiago: Autor. Recuperado de http://www.unesco.org/fileadmin/MULTIMEDIA/FIELD/Santiago/ pdf/Analisis-de-riesgos-de-desastres-en-Chile.pdf

Programa de las Naciones Unidas para el Desarrollo. (2014). Gestión del riesgo de desastres ¿Que hace el PNUD en gestión del riesgo de desastres en América Latina y el Caribe? Panamá: Autor. Recuperado de http://www.latinamerica.undp.org/content/dam/rblac/docs/ Research\%20and\%20Publications/Crisis\%20Prevention\%20and\%20Recovery/Brochure Desastres sp.pdf

Redacción El tiempo (13 de octubre, 2016). Haití, un país que no se repone a los desastres naturales. Internacional. El tiempo. Recuperado de http://www.eltiempo.com/mundo/ latinoamerica/haiti-un-pais-que-no-se-repone-a-los-desastres-naturales-50738

Reyes, A. C. (2012). Implicaciones de la educación en la construcción de una cultura preventiva ante eventos socionaturales. Revista Educare, 16(1), 143-159. Recuperado de http:// revistas.upel.edu.ve/index.php/educare/article/view/754/257

Rivera, M. V. (2007). Saberes y prácticas femeninas en el ordenamiento territorial: Experiencia en el Mirití-Paraná en el Amazonas Colombiano. En L. M. Donato, E. M. Escobar, P. Escobar, A. Pazmiño y A. Ulloa (Eds.), Mujeres indígenas, territorialidad y biodiversidad en el contexto latinoamericano (pp. 109-120). Bogotá: Universidad Nacional de Colombia. Recuperado de http://www2.ecolex.org/server2.php/libcat/docs/LI/MON-080268.pdf 
Secretaría de Medio Ambiente y Recursos Naturales. (2006). Estrategia de educación ambiental para la sustentabilidad en México. Recuperado de http://www.semarnat.gob.mx/ sites/default/files/documentos/educacionambiental/publicaciones/Estrategia\%20 de\%20Educaci\%C3\%B3n\%20Ambiental\%20para\%20la\%20Sustentabilidad\%20-\%20 SEMARNAT\%202006.pdf

Unidad Nacional para la Gestión del Riesgo de Desastres. (2016). Plan nacional de gestión de riesgo de desastres. Una estrategia de desarrollo 2015-2025. Bogotá: Autor. Recuperado de http:// portal.gestiondelriesgo.gov.co/Paginas/Plan-Nacional-Gestion-Riesgo-de-Desastres.aspx 